Psychother Psychosom 2017;86:108-110

DOI: $10.1159 / 000453582$

\section{Emotional Expression Predicts Treatment Outcome in Focal Psychodynamic and Cognitive Behavioural Therapy for Anorexia Nervosa: Findings from the ANTOP Study}

Hans-Christoph Friederich a, b Timo Brockmeyer ${ }^{\mathrm{a}, \mathrm{b}}$, m Beate Wild Gaby Resmark', Martina de Zwaan ${ }^{\mathrm{d}, \mathrm{e}}$, Andreas Dinkelf, Stephan Herpertz ${ }^{\mathrm{g}}$, Markus Burgmer ${ }^{\mathrm{h}}$, Bernd Löwe', Sefik Tagayj, Eva Rothermundk, Almut Zeeck', Stephan Zipfek', Wolfgang Herzog ${ }^{b}$

a Department of Psychosomatic Medicine and Psychotherapy, Medical Faculty, Heinrich Heine University, Düsseldorf, ${ }^{\mathrm{b}}$ Center for Psychosocial Medicine, Department of General Internal Medicine and Psychosomatics, Heidelberg University Hospital, Heidelberg, ' ${ }^{C}$ Department of Psychosomatic Medicine and Psychotherapy, University Hospital Tübingen, Tübingen, ${ }^{\mathrm{d}}$ Department of Psychosomatic Medicine and Psychotherapy, University Hospital Erlangen, Erlangen, ${ }^{e}$ Department of Psychosomatic Medicine and Psychotherapy, Hannover Medical School, Hannover, ${ }^{\mathrm{f}}$ Department of Psychosomatic Medicine and Psychotherapy, Klinikum rechts der Isar, Technical University of Munich, Munich, ${ }^{9}$ Department for Psychosomatic Medicine and Psychotherapy, LWL University Hospital, Ruhr University of Bochum, Bochum, ${ }^{\text {h }}$ Department for Psychosomatic Medicine and Psychotherapy, University Hospital Münster, Münster, 'Institute for Psychosomatic Medicine and Psychotherapy, University Hospital Hamburg-Eppendorf, Hamburg, ${ }^{\mathrm{j}}$ Department of Psychosomatic Medicine and Psychotherapy, LVR Hospital Essen, University of Duisburg-Essen, Essen, ${ }^{\mathrm{k}}$ Department of Psychosomatic Medicine and Psychotherapy, University Hospital of Ulm, Ulm, and 'Department of Psychosomatic Medicine and Psychotherapy, University Hospital Freiburg, Freiburg, Germany; m Section of Eating Disorders, Department of Psychological Medicine, Institute of Psychiatry, Psychology and Neuroscience, King's College London, London, UK

Treatment outcomes for adult anorexia nervosa (AN) patients are generally poor [1]. Examining elements of the therapeutic process could provide helpful new insights into cognitive and behavioural factors associated with a favourable outcome. AN patients typically show strong emotional avoidance, emotion dysregulation, and reduced emotional expression [2,3]. AN behaviours such as dietary restraint may serve as maladaptive mechanisms of emotion regulation by reducing aversive emotional experience [3]. High levels of emotional processing during psychotherapy are usually associated with symptom reduction [4]. This is most likely to occur during mid-treatment when patients rely on the established therapeutic alliance and have learned to deal adaptively with distress. Previous psychotherapy research has demonstrated that emotional processing particularly during mid-treatment predicts favourable treatment outcomes [4]. Our main hypothesis was that greater expression of negative emotions, particularly during midtreatment, is associated with favourable outcomes in AN treatment, independent of the psychotherapeutic approach.

Data were obtained from a multicentre randomised controlled trial comparing 40 sessions of manualised focal psychodynamic therapy (FPT) and enhanced cognitive behaviour therapy (CBT-E) against optimised treatment-as-usual in adult outpatients with AN (for more details of the ANTOP study see [5]). Participants were female, $\geq 18$ years, and had a DSM-IV diagnosis of full-syndrome/ subthreshold AN (BMI 15.0-18.5). Written informed consent was obtained from each participant. Independent research ethics committees approved the study. Participants were randomised to receive either FPT, CBT-E, or optimised treatment-as-usual. Sessions were recorded and stored as audio files only in the FPT and CBT-E condition. Recordings were not available in $21 \%$ of the cases because either the patient or the therapist refused to give informed consent to use the recordings, and in $23 \%$ of the cases recordings were either incomplete or of insufficient quality. The analysis is thus based on the data from 89 patients (FPT: 43; CBTE: 46 ), i.e., $56 \%$ of the original samples, which corresponds to similar studies [6]. These patients did not differ from those excluded regarding BMI, illness duration, eating disorder psychopathology, proportion of restricting subtype, and comorbid disorders at baseline (all $p>0.073$ ). Likewise, FPT and CBT-E participants in this sample did not differ in these variables at baseline, end of treatment, and follow-up (all $p>0.129$ ), except for more common comorbid MDD among CBT-E patients at baseline, $\chi^{2}(1)=5.169$, $p=0.023$. However, patients with and without comorbid MDD did not differ regarding emotional expression in any treatment phase (all $p>0.156)$.

FPT comprised 3 treatment phases: sessions 1-15 focused on therapeutic alliance, pro-anorectic behaviour/beliefs, and self-esteem, sessions 16-32 emphasised emotional experiencing, interpersonal relationships, and their association with problematic eating behaviour, and sessions 33-40 focused on transfer to everyday life, anticipation of treatment termination, and parting. CBT-E comprised several modules: motivation, normalisation of nutrition, creating a formulation, and relapse prevention (mandatory), and cognitive restructuring, mood regulation, social skills, shape concern, and self-esteem (optional).

BMI (calculated as kilograms/metres squared) at end of treatment and at the 12-month follow-up served as the primary outcome. Secondary outcomes were self-reported (Eating Disorders

Hans-Christoph Friederich and Timo Brockmeyer contributed equally to this work.

\section{KARGER}

(C) 2017 S. Karger AG, Basel

E-Mail karger@karger.com

www.karger.com/pps
Dr. Timo Brockmeyer

Department of Psychosomatic Medicine and Psychotherapy

Medical Faculty, Heinrich Heine University Düsseldorf

Bergische Landstrasse 2, DE-40629 Düsseldorf (Germany)

E-Mail timo.brockmeyer@hhu.de 
Inventory-2, EDI-2 [7]) and observer-rated (Structured Interview for Anorexic and Bulimic Syndromes, SIAB-EX [8]) eating disorder psychopathology. Corresponding to previous research, session selection was based on patient ratings of session quality [4]. The highest-rated session according to the Short Inventory for Single Psychotherapy and Counseling [9] (covering motivational clarification/development, active help for problem solving, and therapeutic support) was chosen from the early, middle, and late treatment phase. Additionally, 1 of the initial 3 sessions was chosen alike. When patients rated 2 or more sessions equivalently, 1 of them was chosen randomly. If there were no complete/intact audio recordings available from this session, the session available with the next highest rating was chosen instead. Recordings were available from the initial, early, middle, and late phase from $76,86,81$, and 63 patients, respectively. In line with previous studies, the middle 20 min of sessions (i.e., the "working segment") were selected for analyses [4]. Audio recordings were transcribed and analysed with the Linguistic Inquiry and Word Count (LIWC) [10], a computerised text analysis software that allows for an unobtrusive, non-reactive assessment of naturally occurring verbal emotional expression. The LIWC calculates proportions of words that fall into specific categories (e.g., emotion words).

Sample characteristics, linguistic data, and outcome data are displayed in Table 1. FPT and CBT-E patients did not differ regarding negative emotion expression in any treatment phase (all $p>0.140)$. Across the course of psychotherapy, the amount of negative emotions expressed by patients showed a significant quadratic effect: $F(1,56)=6.10, p=0.017$, indicating an inversed V-shape with greater emotional expression during mid-treatment compared to both the early and late phases (linguistic data from all 3 phases were available from 58 patients). This effect was not moderated by treatment condition: $F(1,56)=3.11, p=0.083$.

A hierarchical multiple regression analysis was conducted with $\mathrm{BMI}$ at end of treatment as the dependent variable. In step 1, BMI at baseline was entered as the predictor into the equation to control for pre-treatment differences in BMI. In step 2, the proportion of negative emotion words expressed by the patient during mid-treatment was added to the model. Negative emotion expression predicted BMI at end of treatment: $B=1.055, \mathrm{SE}=0.346, \beta=0.307, p=0.003$ $\left(R^{2}=0.197\right.$ step $1, p=0.000 ; \Delta R^{2}=0.093$ step $\left.2, p=0.003, n=74\right)$. This effect was not moderated by treatment condition, AN subtype, illness duration, or completer status (all $p>0.131$ ). In an equivalent regression analysis, negative emotion expression predicted BMI at the 12-month follow-up. However, this effect fell just short of significance: $B=0.880, \mathrm{SE}=0.454, \beta=0.215, p=0.057\left(R^{2}=0.099\right.$ step $1, p=0.007 ; \Delta R^{2}=0.046$ step $\left.2, p=0.057, n=73\right)$. The hypothesised causal pathway (greater emotional expression leads to increases in BMI) was further underpinned by the fact that neither BMI after 4 months of treatment (approx. mid-treatment, $p=0.095$ ) nor the change in BMI from baseline to mid-treatment $(p=0.510)$ predicted the magnitude of negative emotion expression at mid-treatment over and above initial negative emotion expression.

Greater negative emotion expression also predicted lower observer-rated eating disorder psychopathology (SIAB-EX) at end of treatment, over and above baseline SIAB-EX scores: $B=-0.199$, $\mathrm{SE}=0.076, \beta=-0.233, p=0.011\left(R^{2}=0.357\right.$ step $1, p<0.000$; $\Delta R^{2}=0.054$ step $\left.2, p=0.011, n=78\right)$. Similarly, it predicted SIABEX total scores at the 12-month follow-up: $B=-0.198, \mathrm{SE}=0.094$, $\beta=-0.226, p=0.040\left(R^{2}=0.245\right.$ step $1, p<0.000 ; \Delta R^{2}=0.050$ step $2, p=0.040, n=65)$. It did not predict self-reported eating disorder
Table 1. Demographic and clinical characteristics, linguistic data, and treatment outcome of the sample $(n=89)$

\section{Demographic and clinical variables}

Age at baseline, years

Illness duration, \% more than 6 years

Anorexia nervosa subtype, $\%$ restricting subtype

Comorbidities, \%

Any

Affective disorder

Anxiety disorder

Somatoform disorder

$27.53 \pm 7.80$

32.6

50.6

34.8

19.1

20.2

3.4

BMI

Baseline

End of treatment $(n=83)$

$16.62 \pm 0.96$

12-month follow-up $(n=81)$

$17.60 \pm 1.78$

$18.27 \pm 2.07$

SIAB-EX total score

Baseline

End of treatment $(n=87)$

$1.05 \pm 0.36$

12-month follow-up $(n=73)$

$0.82 \pm 0.44$

$0.75 \pm 0.46$

EDI-2 total score

Baseline

$265.58 \pm 50.60$

End of treatment $(n=84)$

$246.80 \pm 56.24$

12-month follow-up $(n=71)$

$237.23 \pm 58.65$

Linguistic variables

Total word count

Initial treatment phase $(n=76)$

Early treatment phase $(n=85)$

Mid-treatment phase $(n=80)$

Late treatment phase $(n=62)$

Positive emotion words

Initial treatment phase $(n=76), \%$

Early treatment phase $(n=85), \%$

Mid-treatment phase $(n=80), \%$

Late treatment phase $(n=62), \%$

Negative emotion words

Initial treatment phase $(n=76), \%$

Early treatment phase $(n=85), \%$

Mid-treatment phase $(n=80), \%$

Late treatment phase $(n=62), \%$

$1,936.22 \pm 769.89$

$1,765.92 \pm 744.16$

$1,756.75 \pm 761.16$

$1,699.50 \pm 678.51$

$2.27 \pm 0.60$

$2.32 \pm 0.77$

$2.33 \pm 0.71$

$2.47 \pm 0.65$

$1.23 \pm 0.60$

$1.15 \pm 0.49$

$1.24 \pm 0.51$

$1.05 \pm 0.54$

Data are expressed as means \pm SD unless otherwise indicated. SIAB-EX, Structured Inventory for Anorexic and Bulimic Symptoms; EDI-2, Eating Disorders Inventory-2.

symptoms (EDI-2) either at end of treatment $(p=0.911)$ or at the 12-month follow-up ( $p=0.315)$.

The effects were specific for negative emotion expression, as positive emotion expression during mid-treatment did not predict BMI at end of treatment $(n=74, p=0.082)$ and follow-up $(n=73$, $p=0.258$ ). Likewise, total word count during mid-treatment did not predict treatment outcome (all $p>0.869$ ). Exploratory analysis 
revealed that particularly expressions of sadness $(n=74, B=2.632$, $\mathrm{SE}=0.900, \beta=0.295, p=0.005)$ and anger $(n=74, B=1.753$, $\mathrm{SE}=0.798, \beta=0.227, p=0.031)$ predicted $\mathrm{BMI}$ at end of treatment, whereas anxiety expressions did not $(n=74, p=0.887)$. Similarly, expressions of sadness $(n=73, p=0.059)$ and anger $(n=73, p=$ $0.050)$ predicted BMI at follow-up (the effects, however, fell just short of significance), whereas anxiety expressions $\operatorname{did} \operatorname{not}(n=73$, $p=0.481$ ). The effects of negative emotion expression were specific for mid-treatment (neither negative nor positive emotion expression during the initial, early, or late phase predicted BMI at end of treatment or follow-up, all $p>0.091$ ).

Consistent with our hypothesis, we found that greater expression of negative emotions by patients during mid-treatment predicted favourable treatment outcomes. This is in line with previous psychotherapy research showing strong relationships between emotional processing and treatment outcome [4]. Furthermore, this supports the idea that enhanced emotional processing during psychotherapy for AN may render maladaptive emotion regulation mechanisms (such as dietary restraint) unnecessary. Importantly, these effects were independent from treatment condition, AN subtype, and illness duration, suggesting negative emotion expression constitutes a robust universal action mechanism underlying effective AN treatment. The specific effects of sadness and anger dovetail with reports from AN patients that they perceive these feelings as particularly threatening as they might be rejected by others when expressing them [11].

The study has several limitations. Due to incomplete/noisy recordings and limited consent only half of the original sample could be analysed (note, however, that included and excluded participants did not differ in any sociodemographic or clinical variables). Due to the immense effort the transcription took $(>1,000 \mathrm{~h})$, we furthermore had to restrict our analyses to 4 sessions per patient (accumulating into 303 sessions) and to the middle 20 min of sessions. This may compromise the representativeness of the data. However, this approach is common in psychotherapy research and is assumed to sufficiently reflect typical aspects of the treatment [4, 6]. Quantitative text analysis per se is "blind" to the semantic context and temporal sequence of words and thus cannot provide any information regarding the depth of emotional processing. However, it bears advantages over self-ratings and observer ratings such as high objectivity. Moreover, it is considered a method that could significantly contribute to a better understanding of the active ingredients of psychotherapy [12]. Future studies may also apply more sophisticated text-mining approaches (e.g., $n$-grams, topic models) that include machine-learning procedures [12].
The findings suggest that, independent of treatment approach, therapists should strongly emphasise emotional expression in AN patients, particularly during mid-treatment. Future research should develop and refine treatment modules and techniques that foster emotional expression/processing in AN.

\section{References}

1 Zipfel S, Giel KE, Bulik CM, Hay P, Schmidt U: Anorexia nervosa: aetiology, assessment, and treatment. Lancet Psychiatry 2015;2:1099-1111.

2 Davies H, Wolz I, Leppanen J, Fernandez-Aranda F, Schmidt U, Tchanturia K: Facial expression to emotional stimuli in non-psychotic disorders: a systematic review and meta-analysis. Neurosci Biobehav Rev 2016;64:252-271.

3 Brockmeyer T, Grosse Holtforth M, Bents H, Kämmerer A, Herzog W, Friederich HC: Starvation and emotion regulation in anorexia nervosa. Compr Psychiatry 2012;53:496-501.

4 Watson JC, Bedard DL: Clients' emotional processing in psychotherapy: a comparison between cognitive-behavioral and process-experiential therapies. J Consult Clin Psychol 2006;74:152-159.

5 Zipfel S, Wild B, Gross G, Friederich HC, Teufel M, Schellberg D, Giel KE, de Zwaan M, Dinkel A, Herpertz S, Burgmer M, Löwe B, Tagay S, von Wietersheim J, Zeeck A, Schade-Brittinger C, Schauenburg H, Herzog W; ANTOP study group: Focal psychodynamic therapy, cognitive behaviour therapy, and optimised treatment as usual in outpatients with anorexia nervosa (ANTOP study): randomised controlled trial. Lancet 2014;383:127-137.

6 Loeb KL, Wilson GT, Labouvie E, Pratt EM, Hayaki J, Walsh BT, Agras WS, Fairburn CG: Therapeutic alliance and treatment adherence in two interventions for bulimia nervosa: a study of process and outcome. J Consult Clin Psychol 2005;73:1097-1107.

7 Paul T, Thiel A: Eating Disorder Inventory-2. Deutsche Version. Göttingen, Hogrefe, 2004.

8 Fichter M, Quadflieg N: SIAB-EX. Strukturiertes Interview für anorektische und bulimische Essstörungen nach DSM-IV und ICD-10. Göttingen, Hogrefe, 1999.

9 Krampen G, Wald B: Kurzinstrumente für die Prozessevaluation und adaptive Indikation in der allgemeinen und differentiellen Psychotherapie und Beratung. Diagnostica 2001;47:43-50.

10 Pennebaker JW, Francis ME, Booth RJ: Linguistic Inquiry and Word Count - LIWC 2001. Mahwah, Erlbaum, 2001.

11 Espeset EMS, Gulliksen KS, Nordbø RHS, Skårderud F, Holte A: The link between negative emotions and eating disorder behaviour in patients with anorexia nervosa. Eur Eat Disord Rev 2012;20:451-460.

12 Imel ZE, Steyvers M, Atkins DC: Computational psychotherapy research: scaling up the evaluation of patient-provider interactions. Psychotherapy 2015;52:19-30. 